\title{
On the rate of convergence of the Hamiltonian particle-mesh method
}

\author{
Onno Bokhove ${ }^{1}$, Vladimir Molchanov ${ }^{2}$, Marcel Oliver $^{2}$, and Bob Peeters ${ }^{1}$ \\ 1 Department of Applied Mathematics, University of Twente, The Netherlands \\ 2 School of Engineering and Science, Jacobs University, 28759 Bremen, Germany
}

Summary. The Hamiltonian Particle-Mesh (HPM) method is a particle-in-cell method for compressible fluid flow with Hamiltonian structure. We present a numerical short-time study of the rate of convergence of HPM in terms of its three main governing parameters. We find that the rate of convergence is much better than the best available theoretical estimates. Our results indicate that HPM performs best when the number of particles is on the order of the number of grid cells, the HPM global smoothing kernel has fast decay in Fourier space, and the HPM local interpolation kernel is a cubic spline.

Key words: Hamiltonian particle-mesh method, rate of convergence, numerical tests

\section{Introduction}

The Hamiltonian Particle-Mesh (HPM) method is a particle-in-cell method for compressible fluid flow with the special property that the discrete equations of motion form a Hamiltonian $N$-particle mechanical system. It was originally proposed in the context of the shallow water equations by Frank, Gottwald and Reich [6], and tested on a variety of two-dimensional geophysical flow problems $[3,4,5]$. Moreover, the HPM method was shown to be convergent $[10,11]$ as the number of particles $N$ tends to infinity.

Comparing the HPM method with classical smoothed particle hydrodynamics (SPH) which also possesses a Hamiltonian structure and associated conservation laws $[7,9,13]$, we note that the known convergence results are very similar in the sense that both can be shown to be of order $O\left(N^{2-\varepsilon}\right)$ for any $\varepsilon>0$ provided the underlying kernel functions satisfy certain technical conditions [11]. On the other hand, one time step of the HPM method can be computed in $O(N)$ or $O(N \ln N)$ operations. The computational complexity of SPH is algebraically superlinear in $N$ as the number of interactions per 
particle must grow without bound as $N \rightarrow \infty$. These facts suggest that HPM may be regarded as a "fast" implementation of the SPH algorithm.

In addition to the number of particles $N$, the HPM method has two length scale parameters - the size $\lambda$ of the auxiliary mesh, and a smoothing length scale $\mu$. A priori, these three parameters may be chosen independently. The theory in [11] yields a relation for the scaling of $\lambda$ and $\mu$ as a function of $N$ to achieve a certain rate of convergence; however, it is not known if this relation is optimal. Further, it requires technical conditions on the kernel functions not all of which are met in [6]. Even though satisfactory error behavior is frequently reported, this has not yet been investigated systematically. Finally, it is not known whether the grid itself has a positive impact on the scheme beyond facilitating fast computation of long-range interactions. Thus, the purpose of this paper is to take a very simple, well-controlled numerical setting and study the behavior of HPM as a function of its governing parameters and of the relevant properties of the HPM kernels, and to compare its performance to the classical SPH algorithm.

In this paper, we restrict ourselves to studying the short-time behavior of the HPM method. This corresponds to the analytic results in [11], where the error constants may grow exponentially in time as is typical for general trajectory error estimates for evolution equations. Thus, the Hamiltonian aspects of HPM shall not be considered further. Moreover, we restrict ourselves to two known exact solutions as test cases, namely Burgers' solution in space dimension one and the Iacono cosine vortex in space dimension two. The former is a special solution to the plain irrotational shallow water equations; for the latter, we must include the non-inertial effects of rotation as well as nontrivial bottom topography. Burgers' solution is considered only up to before the time of shock formation. It tests the ability of the particle scheme to cope with increasingly inhomogeneous particle distributions. The cosine vortex is an Eulerian steady state with Lagrangian particle velocities of order one. As such, it serves as a prototype of an optimally well-behaved exact solution which yet poses nontrivial challenges to a particle scheme.

We find that the rate of convergence is much better than the best available theoretical estimates in [11]. Further, our results indicate that HPM performs best when the number of particles is on the order of the number of grid cells, the HPM global smoothing kernel has fast decay in Fourier space, and the HPM local interpolation kernel is a cubic spline.

The outline of the paper is as follows. The continuum shallow water equations and their discretization with the HPM is given in Section 2. Section 3 states a simplified version of the convergence result of [11]. Section 4 introduces the exact special solutions of the shallow water equation that we benchmark against. The numerical results are detailed in Section 5, and the paper concludes with a brief discussion of these results in Section 6 . 


\section{The HPM method for shallow water}

We apply the HPM method to the rotating shallow water equations with bottom topography. On the one hand, the rotating shallow water equations can be seen as a simple example of a barotropic flow. On the other hand, the Coriolis force and bottom topography terms allow for nontrivial exact two-dimensional steady states which we will use as one of our benchmarks.

The continuum equations of motions describe the evolution of a $d$-dimensional velocity field $u=u(x, t)$ and a density field $\rho=\rho(x, t)$ via

$$
\begin{gathered}
\partial_{t} u+u \cdot \nabla u+\mathbb{J} u=-\nabla(\rho+b), \\
\partial_{t} \rho+\nabla \cdot(\rho u)=0,
\end{gathered}
$$

where $\mathbb{J}$ is a zero-order skew-symmetric operator, $b=b(x)$ is a smooth, time-independent function, and $x=\left(x_{1}, \ldots, x_{d}\right)$. When considered on a twodimensional spatial domain, then $u$ describes the evolution of the vertically averaged velocity field, $\rho$ describes the layer depth, and $b$ the spatial variation of the bottom topography of a thin layer of fluid. In the shallow water literature, the layer depth is usually denoted $h$. Here, as in [11], we disregard the physical connotations and write $h$ to denote the numerical approximation to $\rho$. More generally, the forcing term $\nabla \rho$ arises from the choice of barotropic fluid pressure $p(\rho)=\rho^{2} / 2$.

In our experiments, we take $d=1,2$ and supply periodic boundary conditions on $\mathbb{T}^{d} \equiv[-\pi, \pi)^{d}$. When $d=1$, we take $\mathbb{J}=0$; when $d=2$, we use the standard so-called $f$-plane Coriolis term $\mathbb{J} u=\left(-u_{2}, u_{1}\right)$. The physical constant of gravity and the Coriolis constant have been set to unity.

To define the HPM method, we introduce a regular grid with $K$ nodes in each dimension. The locations of the mesh nodes are given by $\left\{x_{\alpha} \equiv \lambda \alpha: \alpha \in\right.$ $\left.\mathbb{G}^{d}\right\}$ on $\mathbb{T}^{d}$, where $\mathbb{G}=\mathbb{Z} \cap[K / 2, K / 2)$ is the index set, always interpreted in modulo $K$ arithmetic, and $\lambda=2 \pi / K$ is the mesh size.

We first define a local partition of unity kernel via a compactly supported shape function $\Psi$. Here, we restrict ourselves to considering tensor-product Bsplines of varying order. We note that the spline of order $r$ satisfies a so-called Strang-Fix condition of order $p=r+1$ which expresses that polynomials of degree less than $p$ can be composed of integer translates of the shape function $\Psi$; it plays a crucial role in the analysis in [11]. All results in the following are labeled by the order $p$ of the Strang-Fix condition used. Once the shape function $\Psi$ is specified, the scaled kernel

$$
\psi_{\lambda}(x)=\lambda^{-d} \Psi(x / \lambda)
$$

and its translates form a periodic partition of unity on the mesh.

Second, we define a global smoothing operator via discrete convolution on the mesh as follows. For a mesh function $h=\left(h_{\alpha}\right)_{\alpha \in \mathbb{G}^{d}}$, the action of the smoothing operator $S_{\lambda, \mu}$ on $h$ at grid node $\alpha \in \mathbb{G}^{d}$ is computed by filtering high frequencies in discrete Fourier space via 


$$
\left(S_{\lambda, \mu} h\right)_{\alpha}=\sum_{\gamma \in \mathbb{G}^{d}} \mathrm{e}^{\mathrm{i} \gamma \cdot x_{\alpha}} \frac{1}{\left(1+|\mu \gamma|^{2}\right)^{q}} \tilde{h}(\gamma),
$$

where $\mu$ defines a global smoothing length scale and

$$
\tilde{h}(\gamma)=\frac{1}{K^{d}} \sum_{\beta \in \mathbb{G}^{d}} \mathrm{e}^{-\mathrm{i} \gamma \cdot x_{\beta}} h_{\beta}
$$

denotes the discrete Fourier transform of $h$. In other words, $S_{\lambda, \mu}$ is a discrete approximation to the $q$-th power of the inverse Helmholtz operator $1-\mu^{2} \Delta$.

The HPM approximation [6] to (1) is a set of ordinary differential equations describing the motion of $N$ fluid particles of mass $m_{k}$ at positions $X_{N}^{k}$, where $k=1, \ldots N$, via

$$
\begin{gathered}
\frac{\mathrm{d}}{\mathrm{d} t} X_{N}^{k}(t)=U_{N}^{k}(t), \\
\frac{\mathrm{d}}{\mathrm{d} t} U_{N}^{k}(t)=-\mathbb{J} U_{N}^{k}(t)-\left.\nabla(\bar{h}(x, t)+\bar{b}(x))\right|_{x=X_{N}^{k}(t)},
\end{gathered}
$$

where the smoothed layer depth $\bar{h}$ and smoothed bottom topography $\bar{b}$ are computed from the finite ensemble of particles in a three-step process. First, we obtain an interpolated layer depth on the grid via

$$
h_{\alpha}(t)=\sum_{k=1}^{N} m_{k} \psi_{\lambda}\left(x_{\alpha}-X_{N}^{k}(t)\right) .
$$

Second, we introduce a smoothed layer depth on the grid,

$$
\bar{h}_{\alpha}(t)=\left(S_{\lambda, \mu} h\right)_{\alpha}
$$

where $h=\left(h_{\alpha}\right)_{\alpha \in \mathbb{G}^{d}}$ denotes the layer depth approximation on the grid. Third, we interpolate the layer depth field from the grid onto the entire domain by using the partition of unity kernels from (2) once more, setting

$$
\bar{h}(x, t)=\lambda^{d} \sum_{\alpha \in \mathbb{G}^{d}} \bar{h}_{\alpha}(t) \psi_{\lambda}\left(x-x_{\alpha}\right) .
$$

Similarly, the bottom topography contribution is computed via

$$
\bar{b}_{\alpha}=\left(S_{\lambda, \mu} b\right)_{\alpha}
$$

where, in abuse of notation, we use $b$ to also denote the topography function evaluated on the grid, and set

$$
\bar{b}(x)=\lambda^{d} \sum_{\alpha \in \mathbb{G}^{d}} \bar{b}_{\alpha} \psi_{\lambda}\left(x-x_{\alpha}\right) .
$$


Expressions (6c) and (7b) can now be analytically differentiated and used on the right hand side of the discrete momentum equation $(5 \mathrm{~b})$. The reason for using the same interpolation kernel $\psi_{\lambda}$ in (6a) and in (6c) is that only then the HPM dynamics has a Hamiltonian structure [6], which can be seen as a direct discrete analog of the parcel Hamiltonian continuum formulation of [2]. This, however, will not play any further role in this paper.

We remark that our scaling of the masses $m_{k}$ in (6a) is that commonly used in the SPH literature. It differs from the definition of $m_{k}$ and $\psi_{\lambda}$ used in [6]. In our scaling, sums over the grid such as appear in (6c) can be read as a Riemann sum approximation of an integral over the torus where $\lambda^{d}$ is the volume of a single cell. Moreover, we can cleanly discuss the SPH limit of HPM, namely the limit that $\lambda \rightarrow 0$ with $N$ and $\mu$ fixed (the $m_{k}$ as defined in [6] become singular in this limit).

In our tests, we initially place $L$ particles in each dimension on a regular particle mesh, so that $N=L^{d}$ and the initial particle positions $X_{N}^{k}$ can be identified, by enumeration, with the initialization mesh points $\left\{X_{\beta} \equiv \Lambda \beta: \beta \in\right.$ $\left.\mathbb{H}^{d}\right\}$ where $\mathbb{H}=1 / 2+\mathbb{Z} \cap[-L / 2, L / 2)$ with particle mesh spacing $\Lambda=2 \pi / L$.

Then, with the same identification between enumeration index $k$ and multiindex $\beta$, we set, at time $t=0, m_{\beta}$ to be an approximation to the mass in cell $\beta$ given by the $d$-dimensional trapezoidal rule

$$
m_{\beta}=\frac{\Lambda^{d}}{2^{d}} \sum_{\gamma} \rho\left(X_{\beta}+\frac{\Lambda}{2} \gamma, 0\right) \approx \int_{X_{\beta}+[-\Lambda / 2, \Lambda / 2]^{d}} \rho(a) \mathrm{d} a,
$$

where the sum ranges over all possible $d$-vectors $\gamma$ whose entries are 1 or -1 .

\section{Theoretical estimates and consequences}

We measure the error in terms of the $L^{2}$-like error functional

$$
\begin{aligned}
Q & =\frac{1}{2} \sum_{k=1}^{N} m_{k}\left|U_{N}^{k}-u\left(X_{N}^{k}\right)\right|^{2}+\frac{\lambda^{d}}{2} \sum_{\alpha \in \mathbb{G}^{d}}\left|\left(S_{\lambda, \mu}^{r} h_{\mathrm{tot}}\right)_{\alpha}-\rho_{\mathrm{tot}}\left(x_{\alpha}\right)\right|^{2} \\
& \equiv Q_{\mathrm{kin}}+Q_{\mathrm{pot}}
\end{aligned}
$$

where $h_{\text {tot }}=h+b$ and $\rho_{\text {tot }}=\rho+b$ are the approximate and exact total layer depths, respectively, and $S_{\lambda, \mu}^{r}$ denotes the convolution square root of $S_{\lambda, \mu}$ which acts, due to the Fourier convolution theorem, on the grid function $h$ via

$$
\left(S_{\lambda, \mu}^{r} h\right)_{\alpha}=\sum_{\gamma \in \mathbb{G}^{d}} \mathrm{e}^{\mathrm{i} \gamma \cdot x_{\alpha}} \frac{1}{\left(1+|\mu \gamma|^{2}\right)^{q / 2}} \tilde{h}(\gamma) .
$$

The error functional can be seen as a direct generalization of the HPM Hamiltonian [6] where the convolution square root arises from symmetrizing the expression for the potential energy. Our error functional is also motivated 
by the one used in [12], where the integral over the layer depth error used there is replaced by its grid-approximation here. Under mild smoothness assumptions, the difference between the two error measures vanishes as $\lambda \rightarrow 0$.

Vanishing of the error functional $Q$ as $N \rightarrow \infty$ corresponds to convergence of the numerical solution of (5-7) to a solution of (1). The following theorem states sufficient conditions for convergence and an associated error bound.

Theorem 1 ([11]). Suppose that the shallow water equations possess a classical solution of class

$$
u, \rho \in C^{1}\left([0, T] ; H^{4+d}\left(\mathbb{T}^{d}\right)\right)
$$

for some $T>0$. Apply the Hamiltonian particle-mesh method with a tensorproduct cardinal B-spline of order $p-1 \geq 2$ as local interpolation kernel and with (3) as the global smoothing operator. Then, with $q>p+d / 2$,

$$
\lambda \sim N^{-\frac{1}{d}}, \quad \text { and } \quad \mu \sim L^{-\frac{p-1}{p+2+d}}
$$

as $N \rightarrow \infty$, there exist constants $C_{1}$ and $C_{2}$ such that

$$
Q(t) \leq C_{1} \mathrm{e}^{C_{2} t} L^{-\frac{2(p-1)}{p+2+d}}
$$

for all $t \in[0, T]$.

A few remarks are in order. First, the theorem was proved for a flat bottom and without rotation; there is, however, no principle obstacle to obtaining a corresponding result in the presence of nontrival topography.

Second, the condition $q>p+d / 2$ excludes the bi-Helmholtz operator used in most of the previous work on HPM. This condition is not sharp, but is technically convenient as it ensures a strict ordering of the various contributions to the total error in the proof of the theorem. Hence, we expect that as $q$ is decreased, the rate of convergence in (13) decreases as well.

Third, the rate of convergence in (13) can be improved to any exponent less than two by choosing a local interpolation kernel which satisfies a sufficiently high order of polynomial reproduction, and subject to choosing $q$ large enough.

Finally, it is possible to let the mesh size $\lambda \rightarrow 0$ while $N$ and $\mu$ remain fixed. Although this limit is not computationally relevant as the computational cost tends to infinity while the accuracy remains finite, it is the limit in which the HPM method tends to the classical SPH algorithm while the sum in the potential energy term of the error functional tends to an integral, so that the error functional converges to the one used by Oelschläger in his proof of convergence of the SPH method [12]. The result is therefore of theoretical interest and can be stated as follows.

Theorem 2 ([11]). In the setting of Theorem 1, suppose that the shallow water equations are solved by the SPH method with periodic kernel given by 
(3) with $q>\max \{3+d / 2, d\}$. Then there exist constants $C_{1}$ and $C_{2}$ such that, letting

$$
\mu \sim N^{-\frac{q-d}{q+2}}
$$

as $N \rightarrow \infty$, we obtain the error bound

$$
Q(t) \leq C_{1} \mathrm{e}^{C_{2} t} N^{-\frac{2(q-d)}{d(q+2)}}
$$

for all $t \in[0, T]$.

In particular, the order of convergence in (15) can be raised to any exponent less than two by choosing $q$ large enough.

\section{Special solutions}

\subsection{Burgers' solution for $d=1$}

We consider the one-dimensional, non-rotating shallow water equations without bottom topography,

$$
\partial_{t} u+u \partial_{x} u=-\partial_{x} \rho \quad \text { and } \quad \partial_{t} \rho+u \partial_{x} \rho=-\rho \partial_{x} u,
$$

for $x \in[-\pi, \pi)$ with periodic boundary conditions. This system has an analytical solution in terms of the function $J(x, t)=K-3 \sqrt{\rho(x, t)}$ satisfying the inviscid Burgers' equation

$$
\partial_{t} J+J \partial_{x} J=0
$$

provided that the Riemann invariant $u+2 \sqrt{\rho}$ initially equals the constant $K$. We choose

$$
\rho(x, 0)=\frac{1}{9}(K+\sin x)^{2} \quad \text { and } \quad u(x, 0)=K-2 \sqrt{\rho(x, 0)}
$$

with $K=3$. This solution develops a discontinuity at the earliest time the characteristics of Burgers' equation intersect, so that

$$
t_{\text {shock }}=-1 / \min \partial_{x} J(x, 0)=1 .
$$

The steepening of the wave in time is shown in Figure 1. Since HPM does not contain shock handling, we are not interested in the behavior at or beyond the singularity. Rather, we perform a multi-parameter study of the behavior of HPM on the time interval $0 \leq t \leq 0.95$, which is close enough to the time of shock solution that the final particle distribution is very nonuniform. This example has also been used in $[1,16]$. 

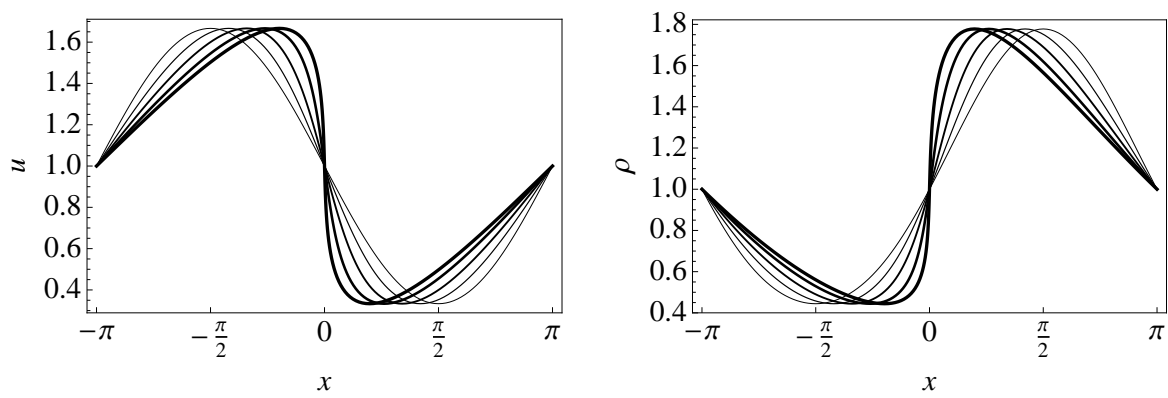

Figure 1. Burgers' solution of the one-dimensional shallow water equations at times $t=0,0.25,0.5,0.75,0.95$.

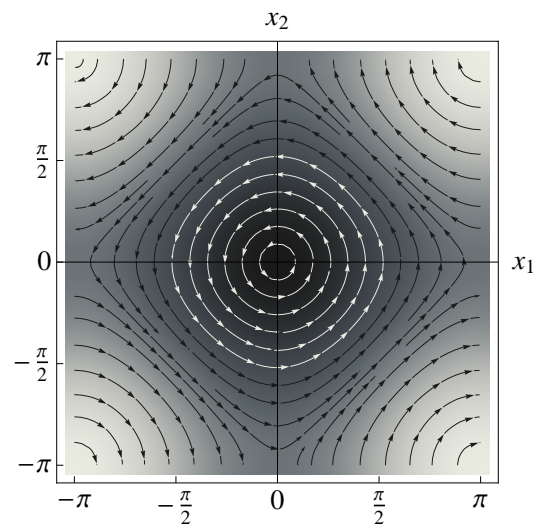

Figure 2. Stream function $\xi$ and stream lines of the cosine vortex (25) with $a=1$ and $c=-1, \rho_{0}=2.5$, and $b_{0}=1$.

\subsection{Cosine vortex over topography for $d=2$}

The cosine vortex in our test case belongs to the family of Iacono vortex solutions [8], special Eulerian steady-state solutions to the two-dimensional shallow water equations. These steady-states are interesting as they pose a nontrivial challenge to Lagrangian methods.

Following [8], we impose that the flow is steady with divergence-free horizontal velocity field. Under these assumptions, the shallow water equations decouple if we consider the Bernoulli function as given. The bottom topography $b$ will then become one of the unknowns.

We begin by defining the relative vorticity $\zeta=\nabla^{\perp} \cdot u \equiv \partial_{1} u_{2}-\partial_{2} u_{1}$ and the Bernoulli function $B(x)=\frac{1}{2}|u(x)|^{2}+\rho(x)+b(x)$, so that the shallow water momentum equations read

$$
(1+\zeta) u^{\perp}+\nabla B=0 .
$$


We then note that the transport stream function $\Psi$, defined via

$$
\rho u=\nabla^{\perp} \Psi,
$$

is in the steady state only a function of $x$, and constant along parcel trajectories. It is known that the potential vorticity $q=(1+\zeta) / \rho$ is materially conserved. Under the steady-state assumption, this implies $q(x)=q(\Psi(x))$. Plugging (21) into (20), we find that $q(\Psi) \nabla \Psi=\nabla B$, so that

$$
\frac{\mathrm{d} B}{\mathrm{~d} \Psi}=q(\Psi)=\frac{1+\zeta}{\rho} .
$$

In the special case that $u$ is divergence free, we can introduce a second stream function $\xi$ via

$$
u=\nabla^{\perp} \xi
$$

Since $\Psi$ is constant along parcel trajectories, $\Psi(x)=\Psi(\xi(x))$. Plugging (23) into (21) yields $\rho \nabla \xi=\nabla \Psi$, so that $\rho$ as function of $\xi$ satisfies $\rho(\xi)=\mathrm{d} \Psi / \mathrm{d} \xi$. Hence, (22) can be written as

$$
\frac{\mathrm{d} B}{\mathrm{~d} \xi}=1+\zeta=1+\Delta \xi .
$$

We now specialize to the class of cosine vortex solutions by setting

$$
\xi(x)=c\left(\cos x_{1}+a \cos x_{2}\right),
$$

where $a$ and $c$ are constants. Consequently, $\nabla^{2} \xi=-\xi$ so that (24) reads $\mathrm{d} B / \mathrm{d} \xi=1-\xi$. Integrating this relation, we obtain

$$
B(\xi)=-\frac{1}{2} \xi^{2}+\xi+B_{0} .
$$

Recalling that

$$
B(\xi)=\frac{1}{2}|\nabla \xi|^{2}+\rho(\xi)+b,
$$

we can determine $\rho(x)=\rho(\xi(x))$ and a consistent bottom profile $b(x)$. Namely, combining (26) and (27), and using (25), we obtain

$$
\rho(\xi(x))+b(x)=-\frac{c^{2}}{2}\left(1+a^{2}+2 a \cos x_{1} \cos x_{2}\right)+\xi(x)+B_{0} .
$$

This equation can be consistently partitioned into two relations $\rho(\xi)=\xi+\rho_{0}$ and $b(x)=-a c^{2} \cos x_{1} \cos x_{2}+b_{0}$, where the constant $\rho_{0}$ is chosen large enough to ensure that $\rho(x)>0$. The cosine vortex with the choice of parameters used in our benchmarks is shown in Figure 2. We remark that the $x_{1}-x_{2}$ plane can be seen as the planar phase space for the dynamics of a single fluid parcel. In this view, $(0,-\pi)$ and $(-\pi, 0)$ and their periodizations are equilibrium points; the line segments $x_{2}= \pm x_{1} \pm \pi$ are heteroclinic connections of these equilibrium points. The vortex appears stable, although a stability analysis using the standard energy-Casimir method [15] is inconclusive. This shall be discussed in more detail in a forthcoming paper. 


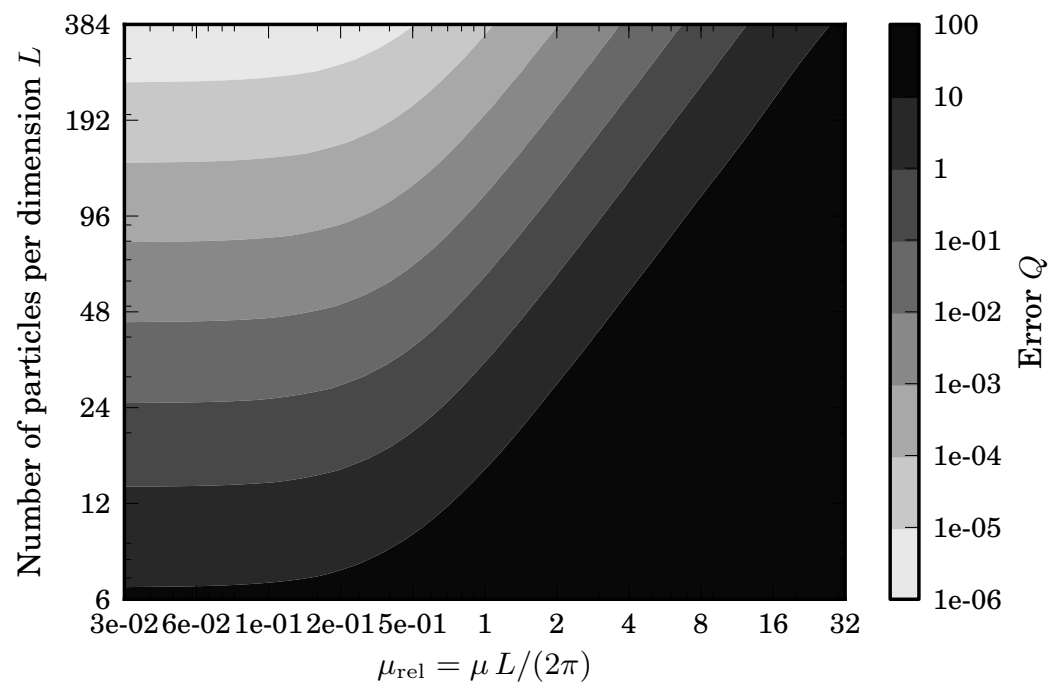

Figure 3. Error levels for the cosine vortex with initially $n=2$ particles per cell per dimension, $q=6, p=4$, and final time $T=0.5$.

\section{Results}

In the following, we present a number of multi-parameter studies of the behavior of the HPM method for the two benchmark cases. To decouple the effects of the mesh size $\lambda$ and the smoothing length $\mu$ as much as possible, we present the results in terms of the relative smoothing

$$
\mu_{\mathrm{rel}}=\mu / \Lambda
$$

where $\Lambda=2 \pi / L$ is the initial inter-particle distance. Thus, for $\mu_{\text {rel }}=1$, the smoothing length scale and the inter-particle distance are comparable. We further let $n$ denote the initial number of particles per cell per dimension, i.e.,

$$
L=n K \quad \text { and } \quad N=L^{d}=n^{d} K^{d} .
$$

As time integrator, we use the standard fourth order explicit Runge-Kutta scheme which is accurate and robust. In our simulations, time integration errors were consistently subdominant for time step $\tau=10^{-4}$. As we are not working on long-time integration, there is no advantage in using a symplectic time integrator as in [6].

\subsection{Optimal global smoothing}

In the first series of benchmarks, we fix the initial number of particles per cell as this is the only regime where the computational effort of the grid operations 


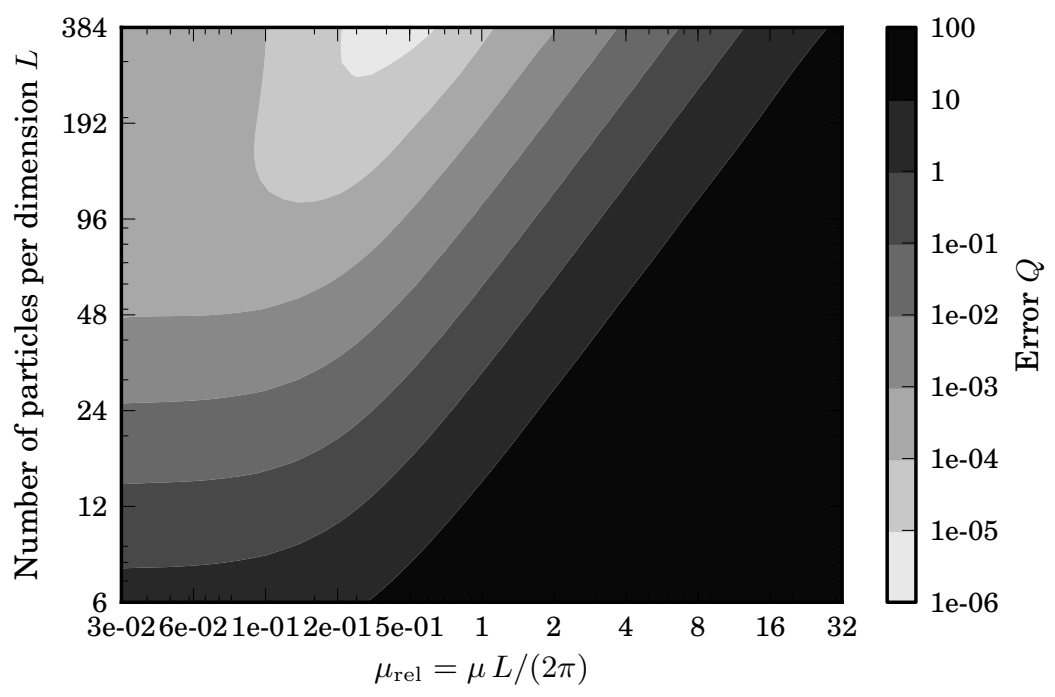

Figure 4. Error levels for the cosine vortex with initially $n=1$ particle per cell per dimension, $q=6, p=4$, and final time $T=0.5$.

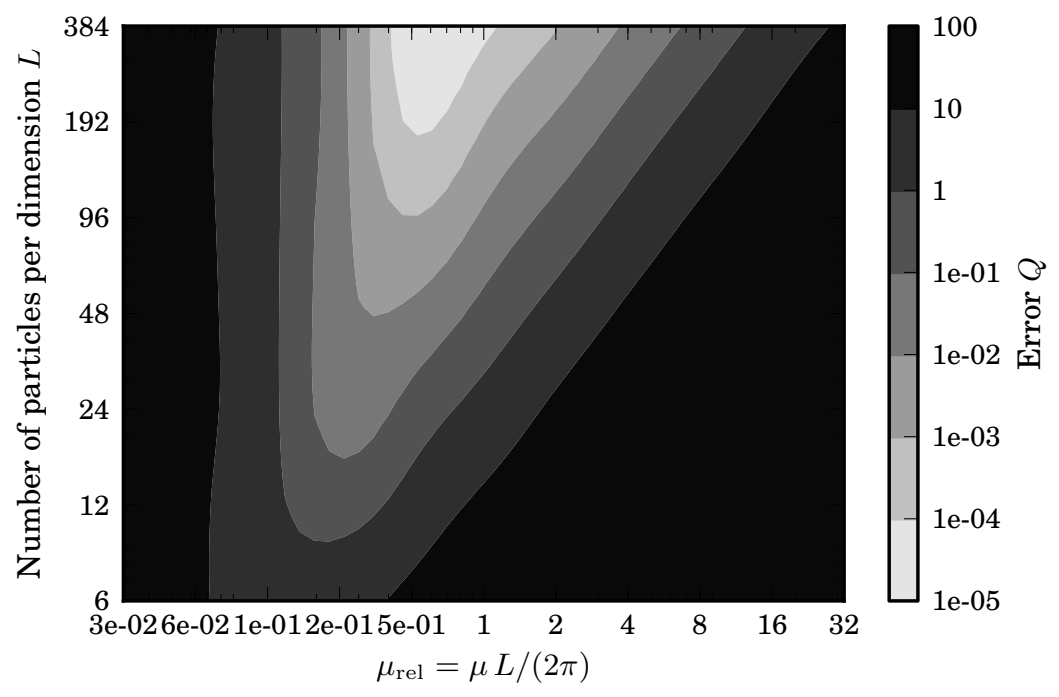

Figure 5. Error levels for the cosine vortex with initially $n=0.5$ particles per cell per dimension, $q=6, p=4$, and final time $T=0.5$. 


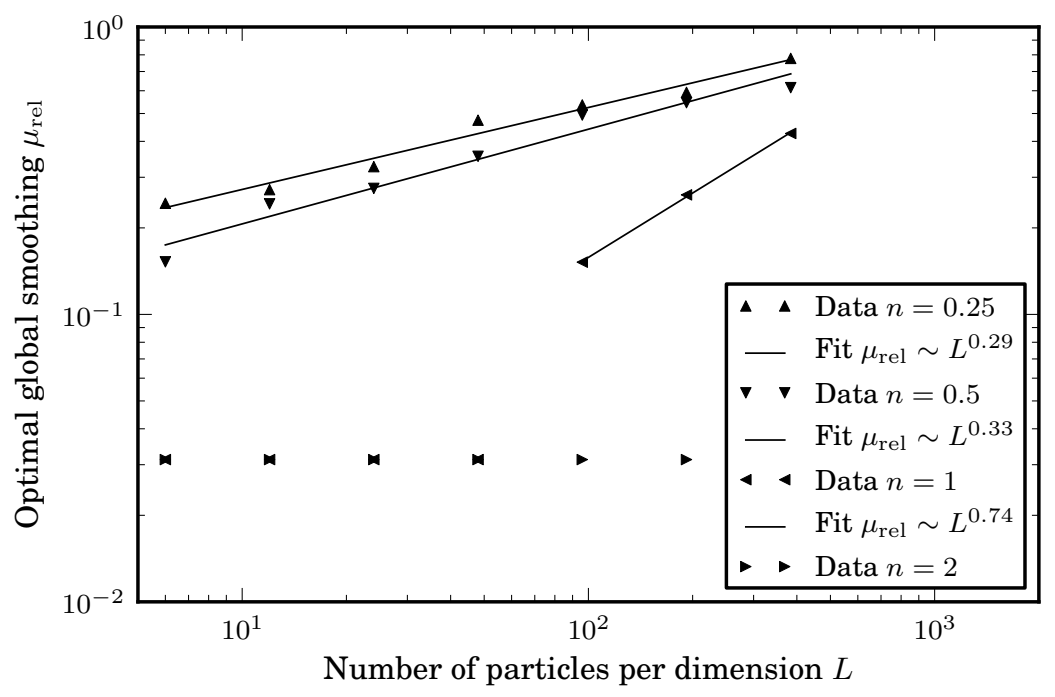

Figure 6. Optimal global smoothing as a function of the number of particles for the cosine vortex with $q=6, p=4$, and $T=0.5$. Note that all of the $n=2$ and some of the $n=1$ data lie exactly at the minimum sampled value for $\mu_{\text {rel }}$. This indicates that global smoothing is not required, i.e., that the true optimal $\mu_{\text {rel }}$ is identically zero.

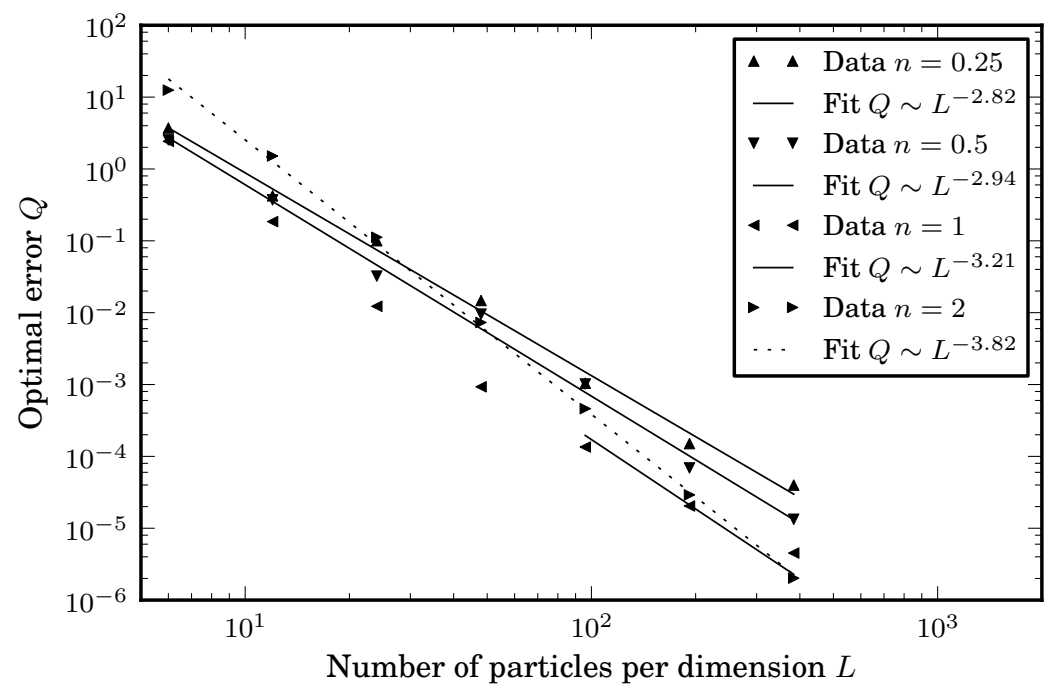

Figure 7. Corresponding minimal error as a function of the number of particles for the cosine vortex with $q=6, p=4$, and $T=0.5$. 
and of the particle-to-grid operations remain asymptotically comparable (up to logarithms). In the first set of tests, we also make sure that the sufficient conditions on the order of the Strang-Fix condition and on the order of the global smoothing under which Theorem 1 asserts convergence of the HPM method are satisfied. In Sections 5.3 and Section 5.4, we later investigate what happens when these conditions are violated.

The qualitative behavior of the error under the change of the number of particles and of the global smoothing length is very similar in all cases. Figures 3 to 5 are examples for the cosine vortex; the behavior for the onedimensional Burgers' solution is very similar. Two features are worth pointing out. First, when the global smoothing is too large, errors rise. In the regime of large global smoothing, contours of constant error have slope 1 in the $\mu_{\mathrm{rel}}-L$ plot; however this is simply saying that $\mu=$ const and therefore to be trivially expected. Second, and more interestingly, when there is a sufficient number of particles per cell, lowest errors are obtained without global smoothing at all. With an increasing number of particles, however, global smoothing appears to become necessary to reduce errors, see Figure 4. We do not know if, as the number of particles increases, global smoothing always becomes necessary, in other words, whether Figure 3 will look like Figure 4 if it were extended toward the larger number of particles regime.

The above indicates that typically, though not always, there is a unique nonzero minimum of the error with respect to the global smoothing for a fixed number of particles. Moreover, these minima lie approximately on a straight line when the coordinate axes are logarithmically scaled, implying that the optimal global smoothing satisfies a power law relationship with the number of particles.

Figure 6 shows the optimal global smoothing for four different initial numbers of particles per cell. The cases $n=2, n=1$, and $n=0.5$ correspond directly to Figure 3, Figure 4, and Figure 5, respectively. For even more initial particles per cell, the behavior is very close to that of $n=2$; for even fewer initial particles per cell, the behavior is very close to that of $n=0.25$. The scaling of the corresponding optimal error is shown in Figure 7.

\subsection{Optimal number of particles per cell}

We can add another level of analysis to the preceding set of simulation data: suppose that for fixed values of the initial number of particles per dimension $L$ and number of particles per cell per dimension $n$ we always choose the optimal global smoothing length scale. How then does the error behave as a function of $L$ and $n$ ? And further, for given $L$, what is the optimal number of particles per cell?

If the cells are too coarse, then clearly we expect the local interpolation error to increase. Whether the error should increase in the opposite extreme, when we refine the mesh without adding more particles, is less obvious. Indeed, Figure 8 shows that this is so for the cosine vortex benchmark, but that there 


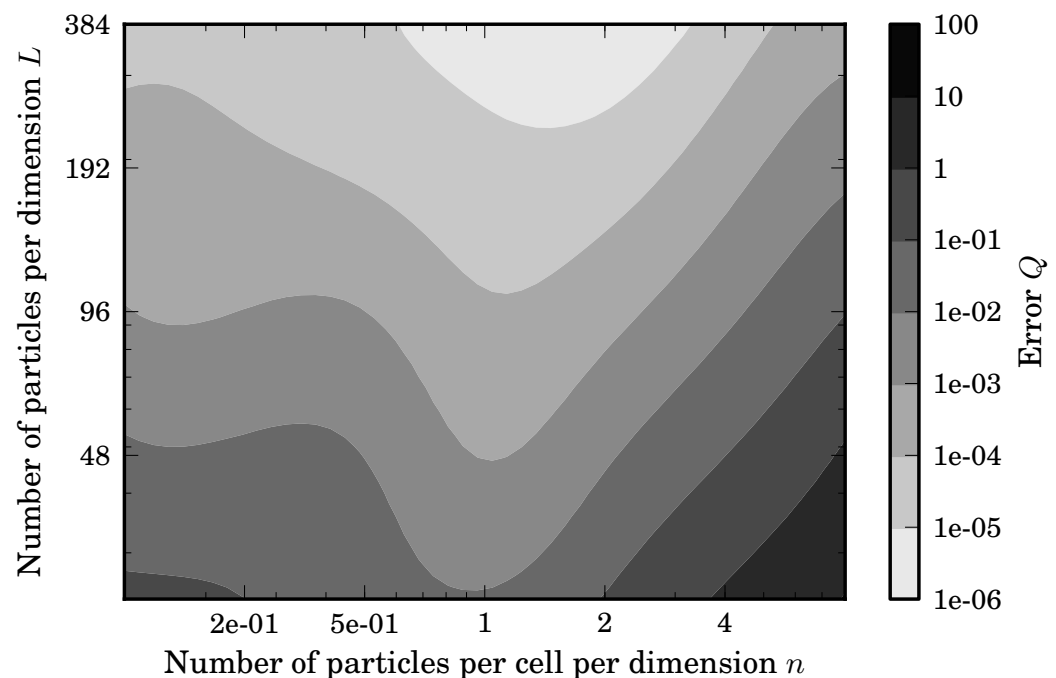

Figure 8. Error levels for the optimally smoothed cosine vortex simulation with $q=6, p=4$, and final time $T=0.5$.

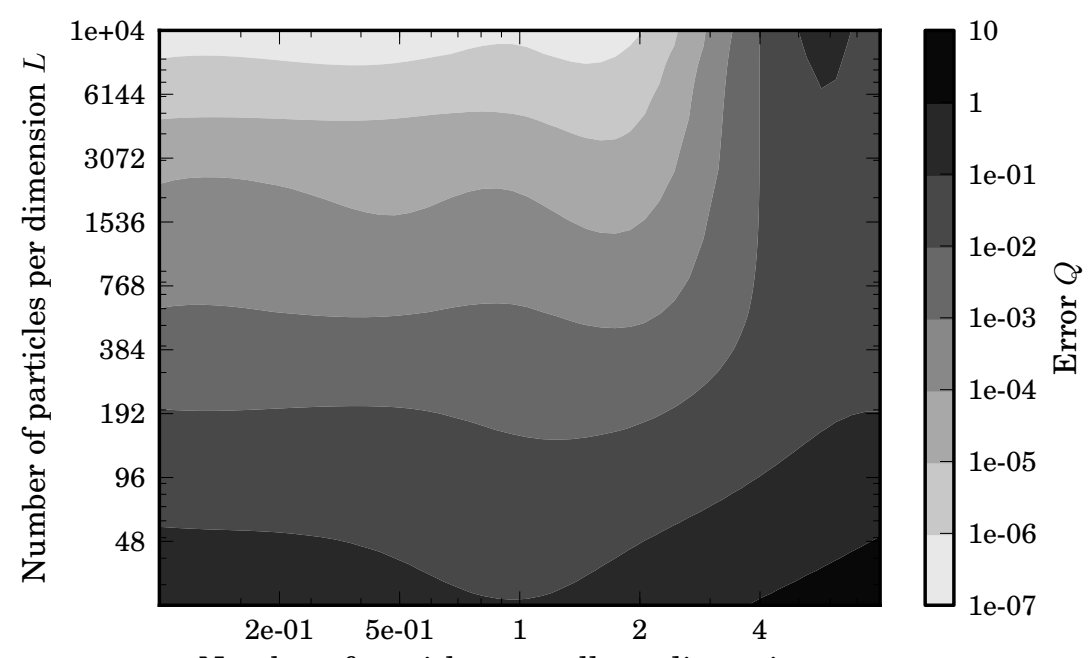

Number of particles per cell per dimension $n$

Figure 9. Error levels for the optimally smoothed simulation of Burgers' solution with $q=6, p=4$, and final time $T=0.95$. 


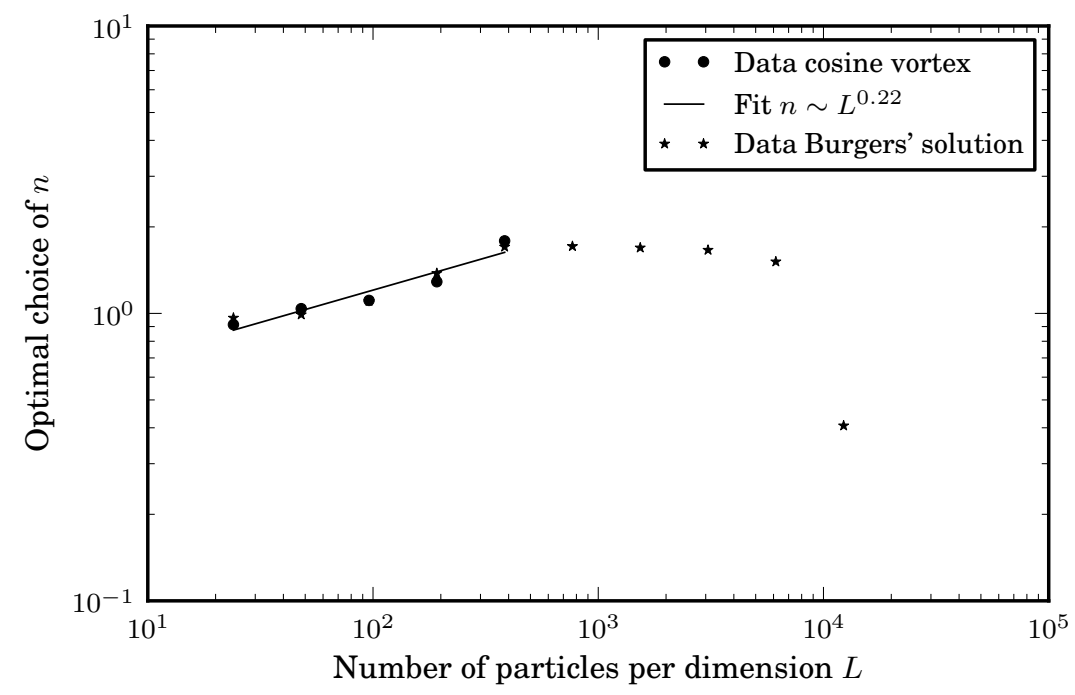

Figure 10. Optimal choice of initial number of particles per cell per dimension $n$ for the optimally smoothed HPM benchmarks shown in Figure 8 and Figure 9.

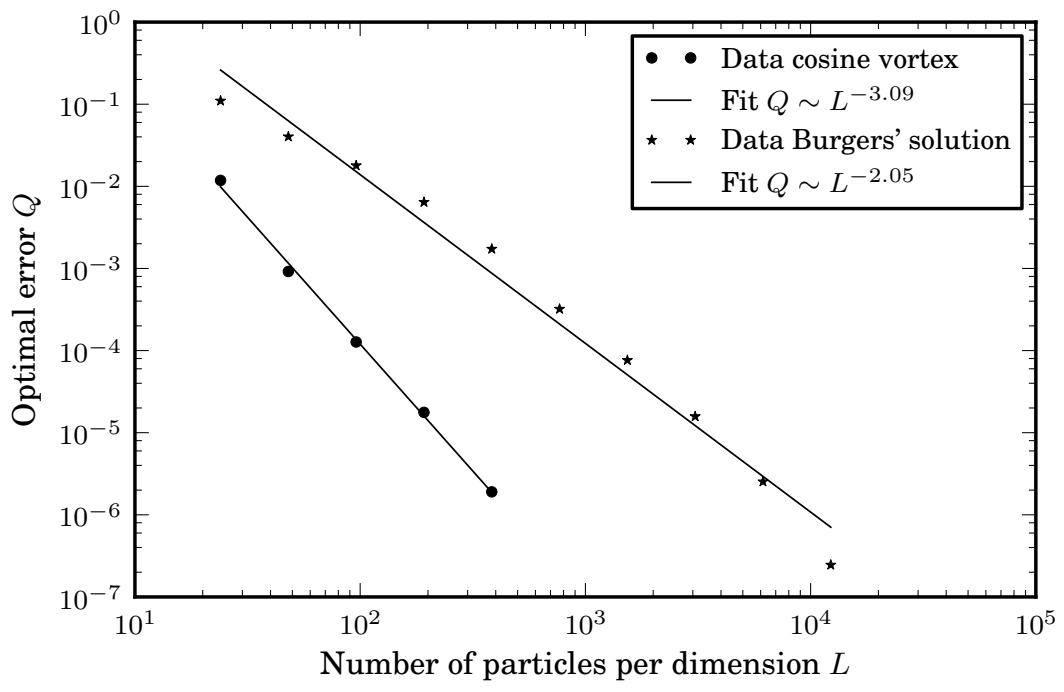

Figure 11. The behavior of the optimal error corresponding to Figure 10. 
is almost no distinct minimum of error for an intermediate number of particles per cell in the Burgers' benchmark, Figure 9.

The optimal number of particles in the vortex case follows very roughly a power law, see Figure 10. For Burgers' solution and and a small number of particles, the scaling behavior is very similar, but Figure 9 makes clear that in this case this behavior is very fragile and indeed breaks down when taking more particles. Yet, the optimal error follows a relatively stable power law in both cases, see Figure 11.

The crucial difference between the two cases is that in the cosine vortex benchmark, the particle distribution remains relatively uniform, while in Burgers' benchmark the particles bunch up toward the right hand side of the domain. In the latter case, HPM cannot do any better than SPH-the SPH limit is characterized by $n \rightarrow 0$ in Figures 8 and 9 . However, in the "good" case when the particles remain approximately uniformly distributed, HPM has a distinct error optimum when the scale particle grid is roughly comparable with that of the HPM grid. This phenomenon was conjectured on theoretical grounds in [11, Remark 1] and is clearly seen in these numerical benchmarks. It also explains why the optimal error scaling in the cosine vortex case is better than for Burgers' solution although generically we expect higher dimensional HPM to do worse than HPM in lower dimensions.

\subsection{The role of the global smoothing order $q$}

Figures 12 and 13 for the cosine vortex and Figures 14 and 15 for Burgers' solution, respectively, show the influence of $q$ on the optimal global smoothing length scale and the corresponding optimal rate of convergence. In the proof of Theorem 1 given in [11], a decay condition of the Fourier symbol of the smoothing kernel implies the requirement $q>p+d / 2$. Numerically, we do not find any indidation that convergence fails when this condition is violated. However, the value of $q$ does feature significantly into the rate of convergence whenever the number of particles per cell drop below unity. (When $n \ll 1$, the optimal smoothing length is zero and the value of $q$ does not enter the computation at all.)

The optimal error in the theoretical estimate is obtained by balancing the influence of the initialization error with that of a dynamical error contribution. This implies that the initialization error, which is essentially dominated by the smoothing error, should scale like the total observed error. Let us therefore look at the scaling of the pure smoothing error. To do so, we recall a wellknown result regarding the $L^{p}$ error for convolution smoothing on $\mathbb{R}^{d}$ [14]. Namely, provided the kernel function satisfies a first order moment condition and some further technical requirements, the smoothing error is of second order in the smoothing length scale $\mu$. Disregarding grid effects, this implies that whenever the optimal smoothing length has scaling $\mu \sim L^{-\kappa}$, the pure smoothing error contribution would scale like

$$
Q_{\text {smooth }} \sim L^{-4 \kappa} \equiv L^{-\gamma_{\text {smooth }}} .
$$




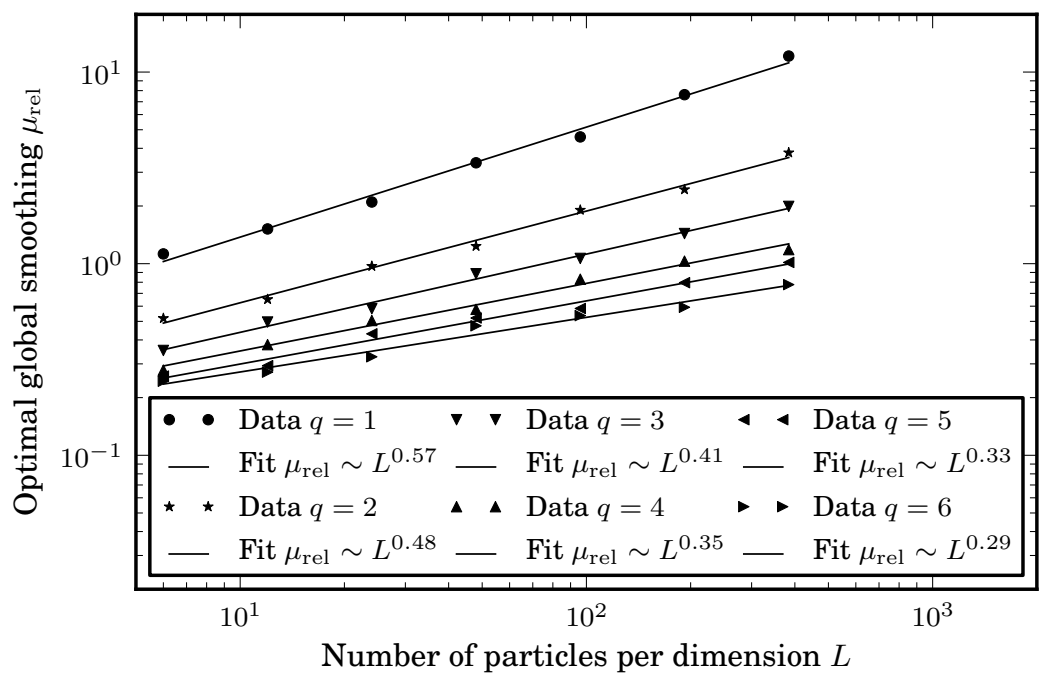

Figure 12. Optimal global smoothing as a function of the number of particles for the cosine vortex with $n=0.25, p=4$, and $T=0.5$.

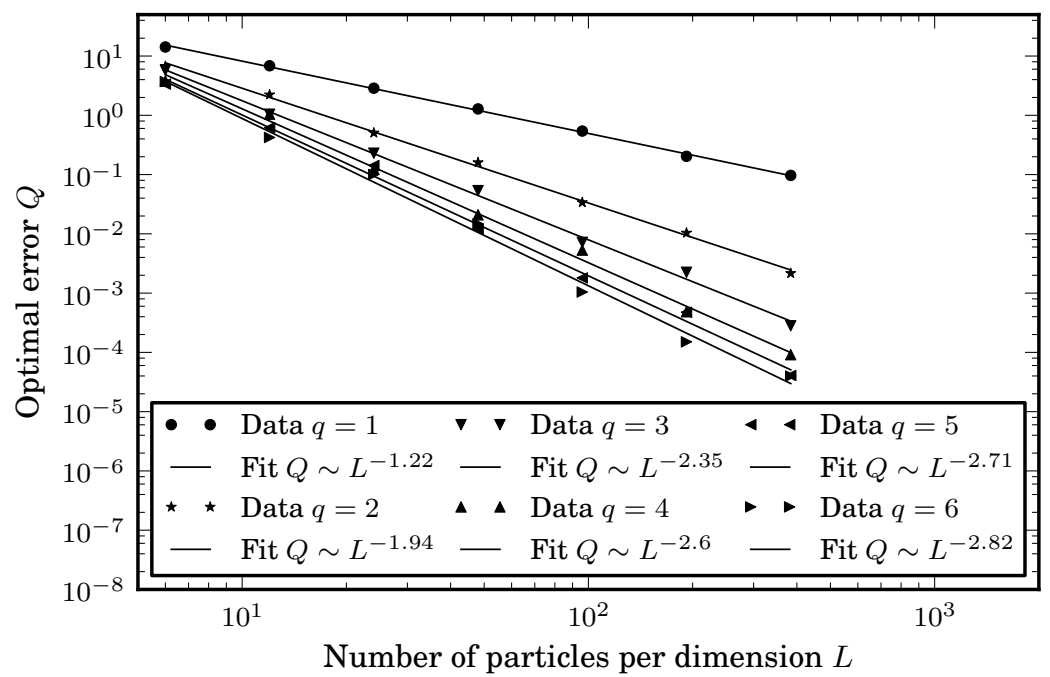

Figure 13. Corresponding minimal error as a function of the number of particles for the cosine vortex with $n=0.25, p=4$, and $T=0.5$. 


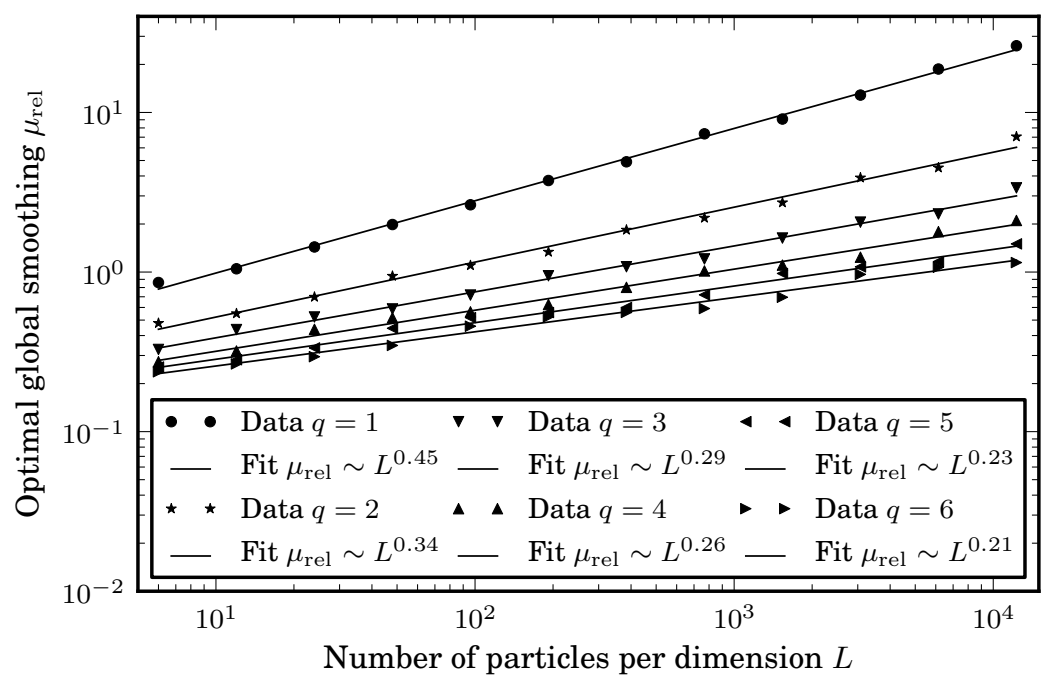

Figure 14. Optimal global smoothing as a function of the number of particles for Burgers' solution with $n=0.25, p=4$, and $T=0.95$.

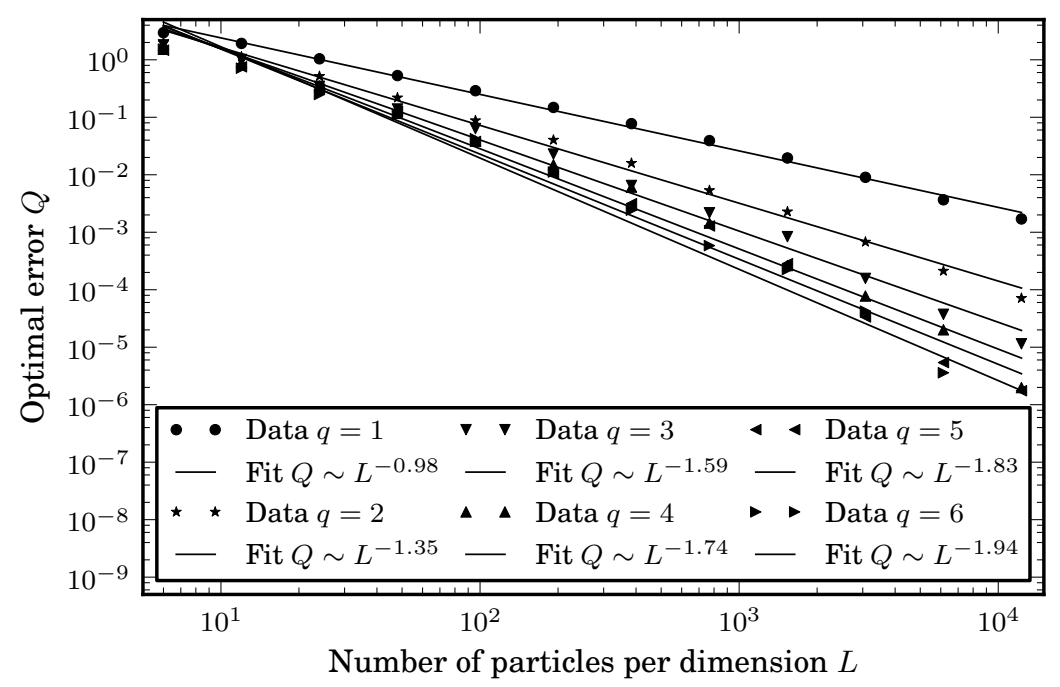

Figure 15. Corresponding minimal error as a function of the number of particles for Burgers' solution with $n=0.25, p=4$, and $T=0.95$. 


\begin{tabular}{lllll}
\hline$q$ & Vortex $\gamma_{\text {act }}$ & Vortex $\gamma_{\text {smooth }}$ & Burgers $\gamma_{\text {act }}$ & Burgers $\gamma_{\text {smooth }}$ \\
\hline 1 & 1.22 & 1.7 & 0.98 & 2.19 \\
2 & 1.94 & 2.09 & 1.35 & 2.62 \\
3 & 2.35 & 2.37 & 1.59 & 2.85 \\
4 & 2.6 & 2.59 & 1.74 & 2.97 \\
5 & 2.71 & 2.68 & 1.83 & 3.08 \\
6 & 2.82 & 2.86 & 1.94 & 3.14 \\
\hline
\end{tabular}

Table 1. Scaling exponents for the error so that $Q \sim L^{-\gamma}$ for the two test cases with cubic splines and $n=0.25$. Compared are actual measured scaling exponents with scaling exponents for pure smoothing error where the smoothing length is given by the actual measured optimal smoothing length.

\begin{tabular}{lllll}
\hline & $\kappa_{\text {theory }}$ & $\kappa_{\text {act }}$ & $\gamma_{\text {theory }}$ & $\gamma_{\text {act }}$ \\
\hline Vortex & 0.38 & 0.71 & 0.75 & 2.82 \\
Burgers & 0.43 & 0.79 & 0.85 & 1.94 \\
\hline
\end{tabular}

Table 2. Scaling exponents for optimal smoothing $\mu \sim L^{-\kappa}$ and corresponding error $Q \sim L^{-\gamma}$ for the two test cases with cubic splines, $q=6$, and $n=0.25$. Notice that $\mu \sim \mu_{\text {rel }} / L$.

Obviously, the HPM dynamics and the grid approximation introduce many further contributions to the total error $Q$ but, by comparing the respective scaling exponents, we shall be able to tell whether the smoothing error is dominant or subdominant.

The results of this analysis are displayed in Table 1, where the behavior for the two test cases is markedly different: for the cosine vortex, the smoothing error is the dominant error contribution except for $q=1,2$ when the order of the operator is way below that required by current theory. We see clearly that, within the expected accuracy, the smoothing error scaling exponent $\gamma_{\text {smooth }}$ provides an upper bound for the actual measured scaling exponent $\gamma_{\text {act }}$. For Burgers' solution, the scaling of the total error is dominated by contributions other than the smoothing error. We conjecture that the particle distribution for the cosine vortex can be seen as a perturbation of a uniform grid for time intervals of order one so that the dynamical error behaves qualitatively similar to the initialization error. For Burgers' solution, on the other hand, the final particle distribution is very nonuniform, so that the dynamical error is distinctly worse than the initialization error; therefore it must be distinctly worse than pure smoothing error scaling. We further believe that the two examples give two extremes of the behavior of HPM under the assumption that the true solution is smooth, and that generic behavior should be within the range covered by these examples. This clearly requires further investigation.

Let us also compare the numerical values of the observed to the theoretically predicted scaling exponents (12) and (13) for cubic splines as local interpolation kernels where $p=4$, read 


$$
\mu \sim L^{-\frac{3}{6+d}} \quad \text { and } \quad Q \sim L^{-\frac{6}{6+d}} .
$$

The comparison for $q=6$ is summarized in Table 2. Clearly, the error bounds given by Theorem 1 are far from sharp. In the case of the cosine vortex where the error behavior is dominated by the smoothing error, and the following interpretation is possible: The scaling exponent of the initialization error is underestimated in [11] by a factor of 2 due to the $L^{1}-L^{\infty}$ splitting used there. As the dynamical error of the cosine vortex behaves qualitatively like the initialization error, this would put the optimal global smoothing scaling exponent off by a factor of 2 , and then again the scaling relation between $\mu$ and $Q$ off by a factor of 2 , so that we might expect an underestimate by a factor of 4 altogether. The data roughly consistent with this explanation, but a more in-depth investigation will be necessary to come to a firm conclusion. In the case of Burgers' solution, the underestimate is not as dramatic as the dominant error contributions are due to other effects which are presumably captured better by the theory.

\subsection{The role of the Strang-Fix order $p$}

To our surprise, the order of the Strang-Fix condition has very little influence on the performance of the SPH method, as Figure 16 illustrates. The support of a linear spline is simply too small to ensure consistent approximation. However, in the quadratic case where $p=3$, the approximation is already quite good with only a slight degradation for large numbers of particles. Moreover, there is no improvement going beyond cubic splines; indeed, the error even rises very slightly, which is likely due to the increased support of the higher order splines and the fact that the examples are taken in a regime where global smoothing for providing long-range interactions is not required.

\section{Conclusion}

We have achieved a comprehensive numerical study of the parameter dependence of the HPM method in a simple, controlled setting. Within the constraints of this setting (looking at the short-time error only, special solutions instead of generic data, and limited parameter ranges), we found that the error behavior as a function of the parameters smoothing length $\mu$, mesh size $\lambda$, and number of particles $N$ is very well characterized by power law dependencies, and thus can be described in simple terms.

We conclude the following. The HPM method performs at least as good as SPH with the same number or particles, although the asymptotic computational cost is much lower. HPM may even perform better than SPH with the same number of particles so long as the particles remain well distributed. Whether this is relevant in the generic situation remains to be studied. 


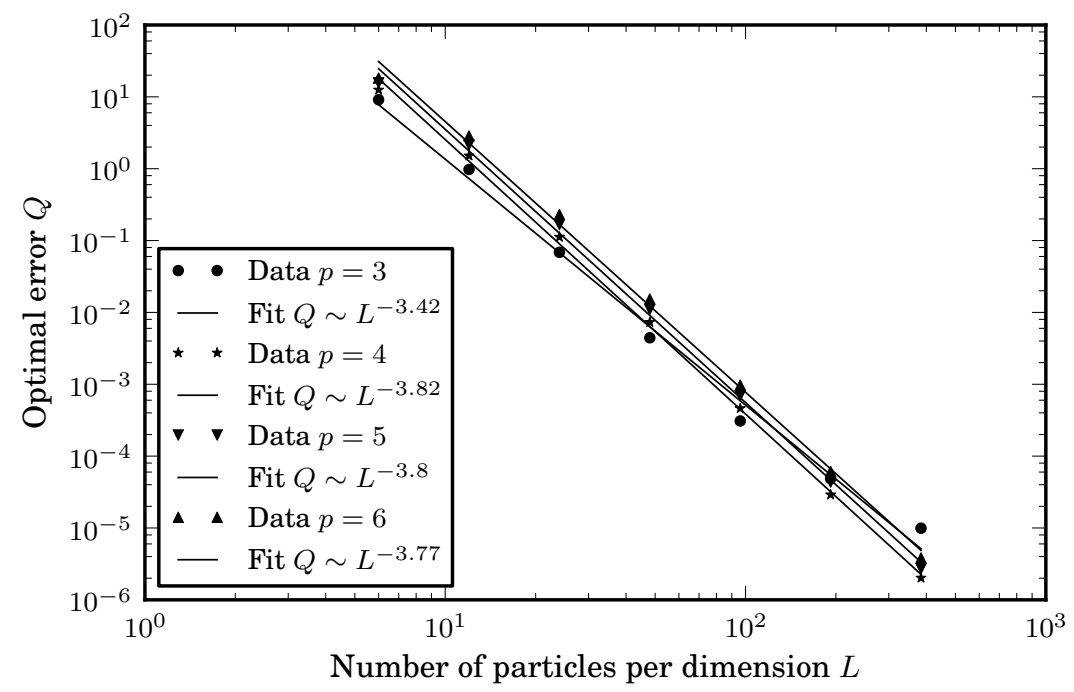

Figure 16. Minimal error as a function of the number of particles for the cosine vortex with $n=2, q=6$, and $T=0.5$. The values $p=3, \ldots, 6$ correspond to quadratic, cubic, quartic, and quintic cardinal B-splines, respectively.

HPM performs best when the number of particles and the number of cells is approximately comparable. In the case when the particle distribution remains favorable, best performance is achieved with a slight asymptotic increase of the number of particles per cell as the number of particles increases. This, however, is likely not relevant in practice, so that a constant number of particles per cell at, or slightly above unity, appears advisable.

The decay exponent $q$ of the global smoothing kernel plays a big role in determining the observed rate of convergence, where larger powers yield better results, although improvements are slight beyond $q=3$ or 4 . However, we do not see a break-down of convergence for low values of $q$ as might be expected from theory.

In contrast, the order of the local interpolation spline plays almost no role for the error behavior. We see a possible slight improvement going from spline order 2 to 3 , but no further benefit beyond that. As the number of interacting grid nodes and the associated computational cost increases with the spline order, the cubic spline appears to be the best choice. Whether an optimally written code with quadratic splines might beat out the cubic version in terms of error per CPU time is beyond what we can assess presently.

We finally remark that our scalings are stated in terms of the number of particles. Since the computational complexity is log-linear in the number of particles, this correlates approximately with scaling in terms of CPU time. 
However, as the discussion of the spline order shows, the constant factors can differ appreciably.

In a forthcoming study, we shall look specifically into the role of the Hamiltonian structure for simulations over longer time scales, and into the behavior with generic data.

\section{Acknowledgments}

V.M. was supported in part through German Science Foundation grant LI1530/6-1. M.O. acknowledges support through German Science Foundation grant OL-155/5-1 and through the European Science Foundation network Harmonic and Complex Analysis and Applications (HCAA). B.P. was supported by the Netherlands Organisation for Scientific Research (NWO) under the grant "Hamiltonian-based numerical methods in forced-dissipative climate prediction".

\section{References}

1. V.R. Ambati and O. Bokhove, Space-time discontinuous Galerkin discretization of rotating shallow water equations, J. Comp. Phys. 225 (2007), 1233-1261.

2. O. Bokhove and M. Oliver, Parcel Eulerian-Lagrangian fluid dynamics for rotating geophysical flows, Proc. Roy. Soc. A 462 (2006), 2563-2573.

3. C.J. Cotter, J. Frank, and S. Reich, Hamiltonian particle-mesh method for twolayer shallow-water equations subject to the rigid-lid approximation, SIAM J. Appl. Dyn. Syst. 3 (2004), 69-83.

4. J. Frank and S. Reich, Conservation properties of smoothed particle hydrodynamics applied to the shallow water equations, BIT 43 (2003), 40-54.

5. J. Frank and S. Reich, The Hamiltonian particle-mesh method for the spherical shallow water equations, Atmos. Sci. Let. 5 (2004), 89-95.

6. J. Frank, G. Gottwald, and S. Reich, A Hamiltonian particle-mesh method for the rotating shallow water equations, Meshfree Methods for Partial Differential Equations (M. Griebel and M.A. Schweitzer, eds.), Lecture Notes in Computational Science and Engineering Vol. 26, Springer, 2002, pp. 131-142.

7. R.A. Gingold and J.J. Monaghan, Smoothed particle hydrodynamics: theory and application to non-spherical stars, Mon. Not. R. Astron. Soc. 181 (1977), 375389.

8. R. Iacono, Analytic solution to the shallow water equations, Phys. Rev. E 72 (2005), 017302.

9. L.B. Lucy, A numerical approach to the testing of the fission hypothesis, Astrophys. J. 82 (1977), 1013.

10. V. Molchanov, "Particle-Mesh and Meshless Methods for a Class of Barotropic Fluids," PhD thesis, Jacobs University, 2008.

11. V. Molchanov and M. Oliver, Convergence of the Hamiltonian particle-mesh method for barotropic fluid flow, Math. Comp., in press. 
12. K. Oelschläger, On the connection between Hamiltonian many-particle systems and the hydrodynamical equations, Arch. Rational Mech. Anal. 115 (1991), 297310.

13. D.J. Price, Smoothed particle hydrodynamics and magnetohydrodynamics, J. Comput. Phys. 231 (2012), 759-794.

14. P. Raviart, An analysis of particle methods, Numerical Methods in Fluid Dynamics (F. Brezzi, ed.), Lecture Notes in Mathematics Vol. 1127, Springer, 1985, pp. 243-324.

15. P. Ripa, General stability conditions for a multi-layer model, J. Fluid. Mech. 222 (1991), 119-137.

16. P. Tassi, O. Bokhove, and C. Vionnet, Space-discontinuous Galerkin method for shallow water flows - kinetic and HLLC flux, and potential vorticity generation, Adv. Water Res. 30 (2007), 998-1015. 